\title{
Mars Ascent Vehicle Test Requirements and Terrestrial Validation
}

\author{
John W. Dankanich \\ Gray Research, Inc. \\ 21000 Brookpark Rd. M/S 77-4 \\ Cleveland, $\mathrm{OH} 44135$ \\ 216-433-5356 \\ John.Dankanich@,nasa.gov \\ Henry M. Cathey \\ New Mexico State University, Wallops, VA, 23337 \\ Ian J. Dux \\ NASA Glenn Research Center, Cleveland, OH 44135 \\ David A. Smith \\ Wyle Information Systems, LLC, Cleveland, OH, 44135
}

\begin{abstract}
The Mars robotic sample return mission has been a potential flagship mission for NASA's science mission directorate for decades. The Mars Exploration Program and the planetary science decadal survey have highlighted both the science return of the Mars Sample Return mission, but also the need for risk reduction through technology development. One of the critical elements of the MSR mission is the Mars Ascent Vehicle, which must launch the sample from the surface of Mars and place it into low Mars orbit. The MAV has significant challenges to overcome due to the Martian environments and the Entry Descent and Landing system constraints. Launch vehicles typically have a relatively low success probability for early flights, and a thorough system level validation is warranted. The MAV flight environments are challenging and in some cases impossible to replicate terrestrially. The expected MAV environments have been evaluated and a first look of potential system test options has been explored. The terrestrial flight requirements and potential validation options are presented herein ${ }^{1,2}$
\end{abstract}

\section{TABLE OF CONTENTS}

1. INTRODUCTION.

2. MAV SYSTEM REQUIREMENTS.. . .1

3. MAV TEST OPTIONS...........................................П.....6

8. CONCluSiONS ....................................................而.......12

REFERENCES ........................................................而......12

BIOGRAPHIES .............................................................. سn....13

\section{INTRODUCTION}

For decades, NASA and the science community have been planning for the robotic Mars Sample Return (MSR) mission. One of the highest risk elements of the MSR campaign perceived or real, is the Mars Ascent Vehicle
(MAV). The MAV is essentially a small launch vehicle that must be erected and launched from a landed platform. New launch vehicles have a relatively low probability of mission success with historical rates of 59 percent and 77 percent for first and second launches respectively. The MAV is likely to be a launch vehicle with derived heritage for the key subsystems, but even derived vehicles have only demonstrated a historic success rate of 73 percent for their first flights. Based on these statistics, is has been recommended that NASA perform at least two terrestrial integrated flight tests prior to the MSR mission PDR.

To implement the MAV technology development and terrestrial flight tests, NASA's In-Space Propulsion Technology (ISPT) Project has initiated MAV investments. The objective of the ISPT project is to develop technologies to enhance or enable planetary science missions by increasing performance while reducing mission cost and risk; with an emphasis on sample return technologies. The ISPT project is establishing the flight system requirements, defining system interfaces, and is evaluating test options. The ISPT project also awarded three industry contracts to begin the MAV flight system design and develop and qualify the propulsion subsystem in October 2010. The baseline MAV project plan will mature the subsystem technologies and then integrate the MAV for multiple terrestrial flight tests that must occur prior to the MSR lander mission preliminary design review (PDR).

The baseline MSR mission architecture has been established and will begin implementation starting with the joint NASA / ESA 2018 mission. The 2018 mission includes the MAX-C Rover to collect and cache samples to be returned. The MSR Orbiter and Lander missions are planned for 2022 and 2024 respectively. The architecture is shown in Figure 1.

\footnotetext{
${ }^{1}$ 978-1-4244-7351-9/11/\$26.00 (C2011 IEEE.

2 IEEEAC paper \#1820, Version 2, Updated January 3, 2011
} 


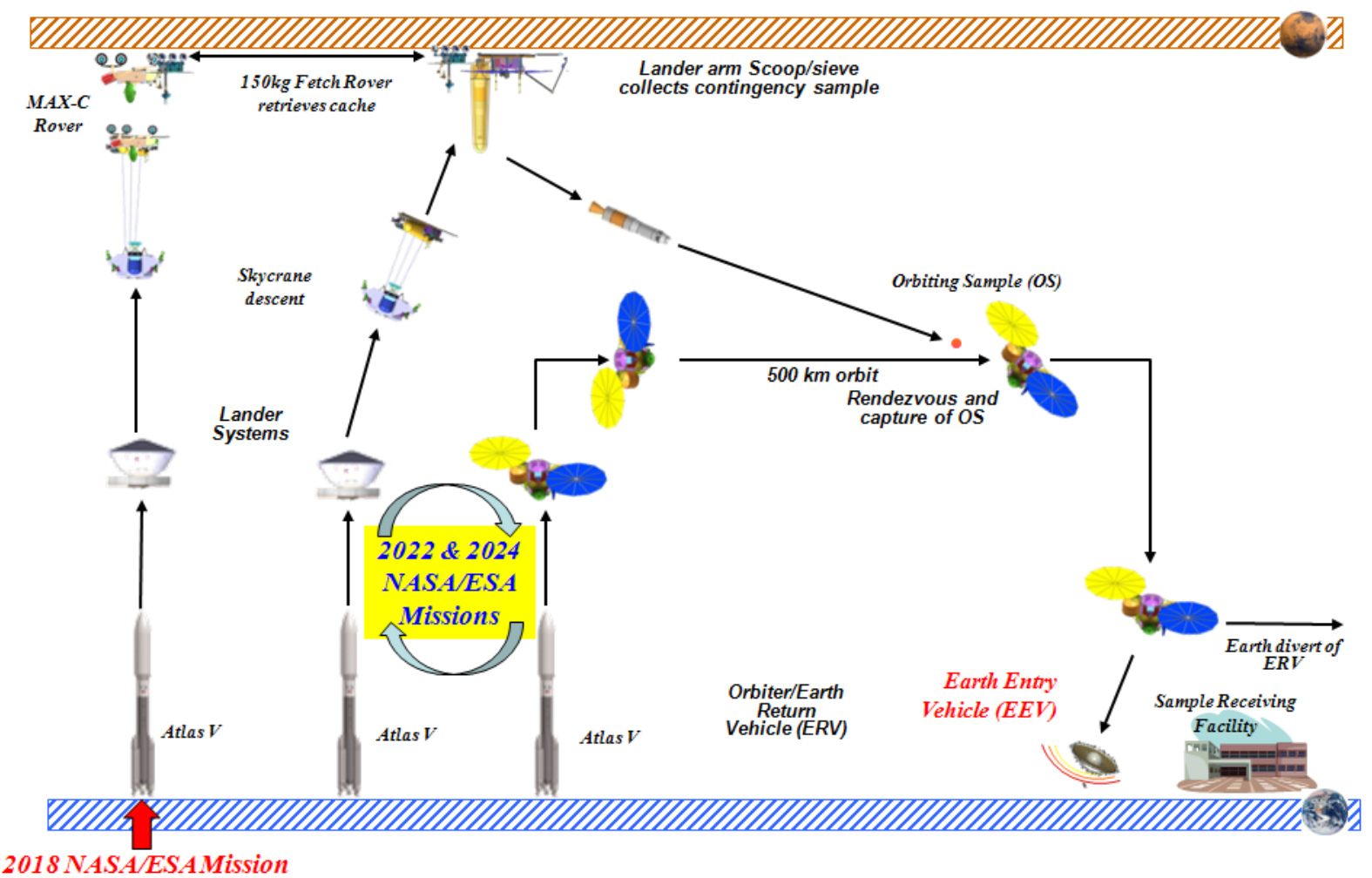

Figure 1 - Baseline MSR Architecture.

The MSR campaign drives the need date for the MAV technology development and test program schedule. A tentative schedule for the MAV project is shown in Figure 2. In order to have the system validated with sufficient schedule margin to allow a 2024 lander mission PDR, the flight tests are scheduled to occur in 2018. In order to establish a credible development schedule and test plan, the test requirements and potential options have been explored. The preliminary test requirements and first look at potential test options are presented.

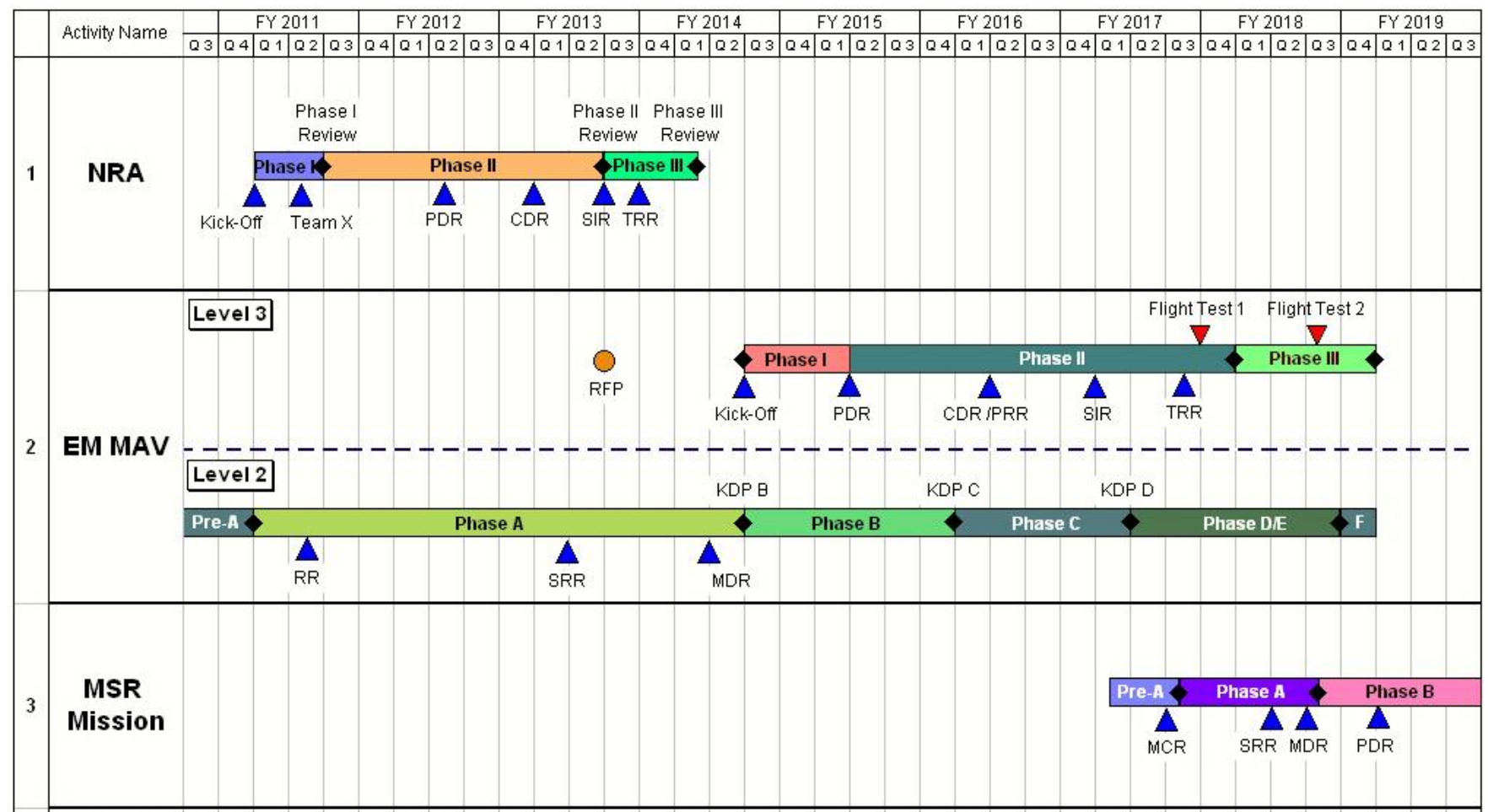

Figure 2 - Notional MAV Development Schedule. 


\section{MAV SYSTEM REQUIREMENTS}

The MAV level 1 requirements are that the vehicle shall deliver a $5 \mathrm{~kg}$ orbiting sample (OS) into a $500 \mathrm{~km}$ circular orbit at Mars. Specifically:

Launch Site: $\quad 15^{\circ} \mathrm{S}-25^{\circ} \mathrm{N}$ Latitude (TBD)

$-1 \mathrm{~km}$ elevation (MOLA aeroid)

Target Orbit: $\quad 500 \mathrm{~km}$ Altitude $+/-100 \mathrm{~km}$ dispersions $45^{\circ}$ inclination $+/-0.2^{\circ}$

The MAV must also be single fault tolerant and have the ability to generate and transmit both real-time and recorded engineering data to an orbiter with enough fidelity to discern root cause of decreased performance or failure. Because the MAV is baselined to be placed on the surface of Mars using the MSL SkyCrane Entry Descent and Landing (EDL) system, there are significant physical and environmental constraints placed the MAV and supporting systems. The MAV must also able to survive on the surface of Mars quiescent for up to six months before completing its primary mission.

\section{Performance Requirements}

The detailed performance requirements for the MAV are still TBD, as the specific subsystems are yet to be selected. Potential test requirements have been derived from baseline MAV designs or subsystem assumptions, so the necessary test parameters can be better understood. For the purpose of this paper, the performance requirements are for the MAV launch phase. In most cases, performance will be validated at the component and subsystem level. The key top-level performance requirements to be validated during integrated test include:

Primary Propulsion-The primary propulsion systems sized based on the system dry mass, launch elevation, azimuth, etc. A wide range of trades have been conducted to envelope the expected performance requirement [1]. The baseline solution is a two-stage to orbit (TSTO) MAV launch profile. A notional launch profile is illustrated in Figure 3. The vehicle launches and starts a commanded pitch over maneuver after clearing the launch tube, first stage burnout occurs followed by staging and a long coast period before performing the second stage circularization maneuver. The duration of the first stage burn is highly dependent on the propulsion system characteristics.

Based on a 45 degree launch elevation and within 20 degrees of nominal launch assumptions, the performance of the first and second stages are 2,662 m/s and 1,486 m/s respectively.

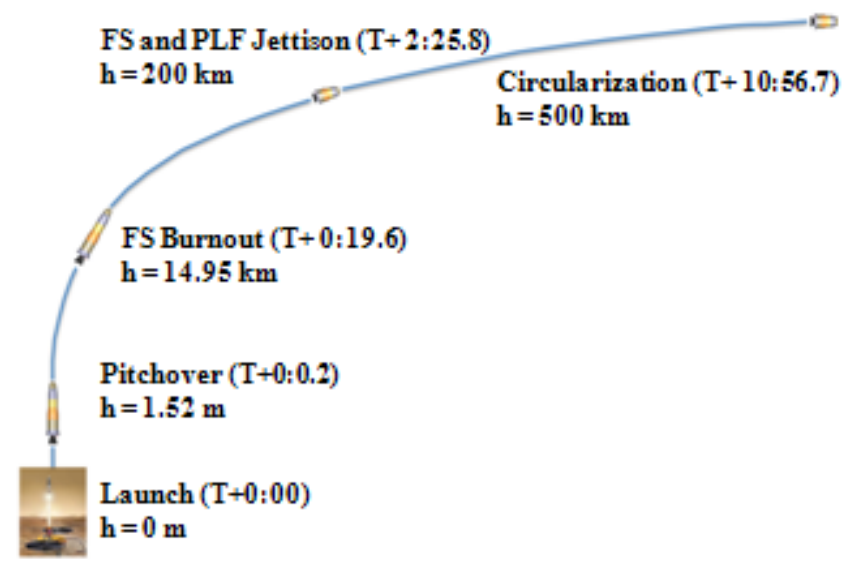

Figure 3 - Notional TSTO Launch Profile.

RCS Propulsion and TVC-The reaction control system (RCS) requirements are also TBD at this stage, but the baseline assumes Thrust Vector Control (TVC) with nozzle actuators during motor firing and RCS during the coast phase. The TVC system is assumed to be able to provide 5 $\mathrm{deg} / \mathrm{s}$ pitch over rates and the RCS system provides 107 Nms of pitch and yaw control and $38 \mathrm{Nms}$ of roll control. RCS and TVC estimates are based only on 3 DOF optimization with 6 DOF simulations to be completed. The test must validate maximum control rates and control authority.

$G N \& C$ - The baseline MAV guidance scheme is for the vehicle to follow an open loop attitude profile throughout the first stage burn as well as the coast period before the second stage circularization maneuver. A closed loop algorithm will be employed during the second stage burn to correct for accumulated trajectory errors prior to the second maneuver. The performance requirement to be validated will be final orbit injection accuracies.

Separation Events-The primary objective from an integrated test is to validate the successful systems sequence of events. Staging of the first stage is best validated by test. The potential for re-contact is real and has been experienced on several early launch system flight attempts. Because of the long coast period between first stage shutdown and second stage ignition with negligible performance impact of stage separation timing, the potential for re-contact can be minimized, but successful staging should be validated by test.

Telemetry and Communications - The MAV has a level 1 requirement to generate and transmit engineering data to discern potential failure modes. To meet this requirement, the MAV will have significant instrumentation, 
accelerometers, pressure transducers, temperature sensors, etc. It is assumed the MAV must then transmit the data at $38.4 \mathrm{kbps}$ to an orbiter with a maximum slant range of $2,400 \mathrm{~km}$ using an Electra [2] derived UHF transfer throughout the MAV launch phase from launch minus 30 seconds through OS deployment plus one minute.

\section{Environmental Requirements}

For the purpose of this study, it is assumed that the MAV will have external systems for thermal control, support structure, power, etc. prior to initiating the launch sequence. The MAV is expected to be at a minimum temperature of $40^{\circ} \mathrm{C}$, but most likely at an elevated temperature of $4^{\circ} \mathrm{C}$ (TBD) at launch. The MAV will have to meet environmental

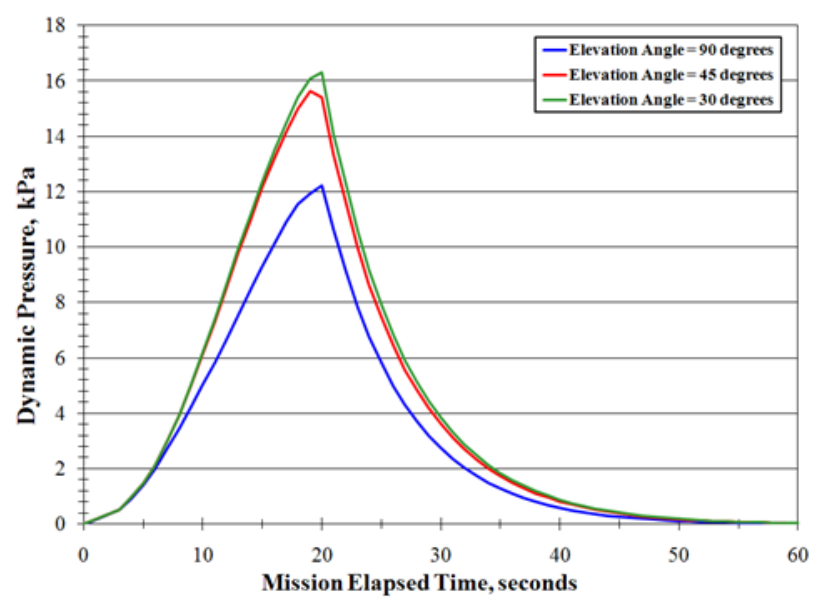

requirements for several phases of the mission. For environmental requirements during the launch phase of the MAV mission, the MAV must meet the performance requirements given the atmospheric, thermal and gravity environments on Mars.

The most critical environments for the MAV launch phase

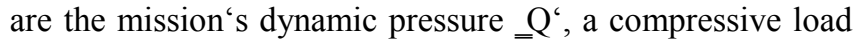
indicator, and the Q-alpha, the bending load indicator. The maximum dynamic pressure of an ATK Star solid motor is significantly higher than an optimal thrust, potentially liquid, solid motor. The maximum Q and Q-alpha for high thrust solid and optimal thrust, assumed liquid, propulsion is illustrated in Figures 4 and 5. The maximum values of $\mathrm{Q}$ and Q-alpha are functions of both the launch elevation and the thrust. For a $45^{\circ}$ launch elevation on Mars, the expected maximum dynamic pressures will be $457 \mathrm{psf}$ and $120 \mathrm{psf}$ for the high thrust and optimal thrust cases respectively. The bending load parameters are 54 psf-deg and 169 psf-deg for the high thrust and optimal thrust cases respectively.

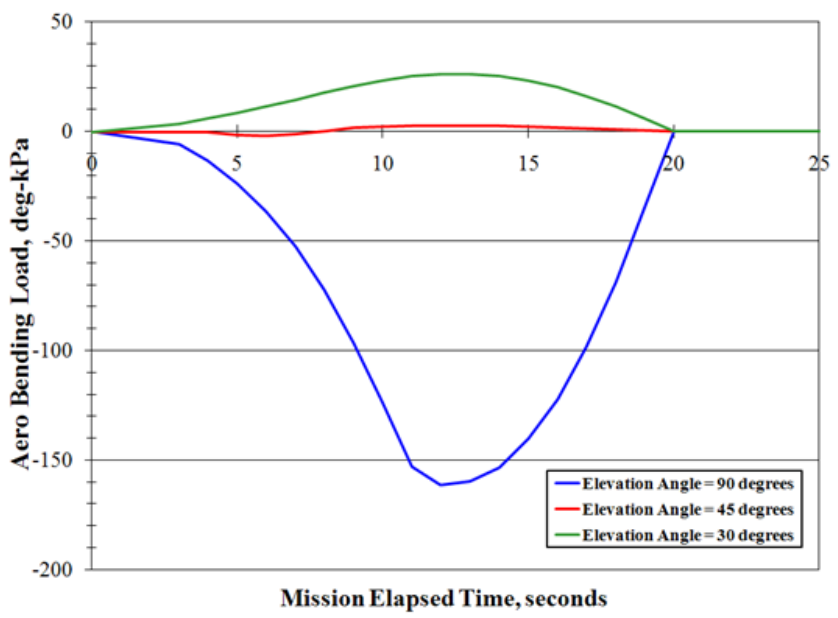

Figure 4 - Q (Dynamic Pressure) and Q-Alpha (Aerodynamic Bending Load) for high thrust TSTO profile.
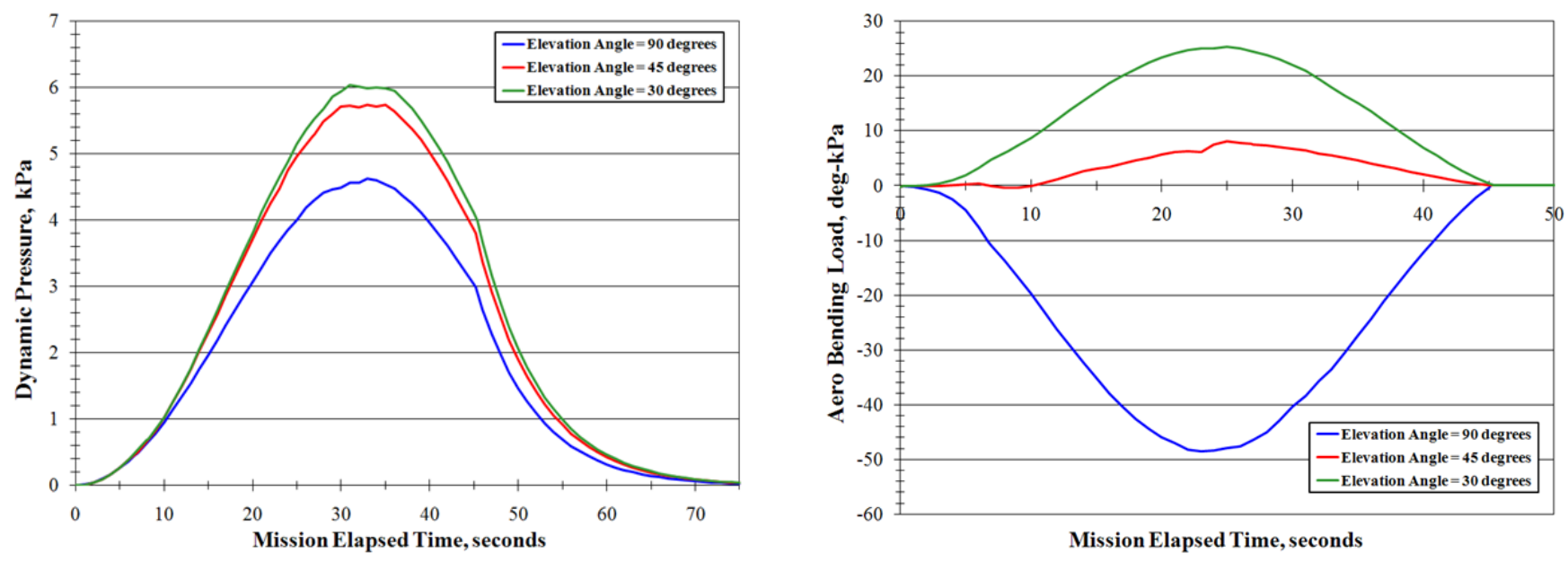

Figure 5 - Q (Dynamic Pressure) and Q-Alpha (Aerodynamic Bending Load) for optimal thrust TSTO profile. 


\section{MAV TEST OPTIONS}

The complexity of the robotic Mars Sample Return mission, or risk tolerance of any NASA flagship mission, necessitates a robust test program. The MAV presents several unique challenges of a vehicle test program, but they must be addressed to maximize the probability of mission success.

\section{Altitude Test Options}

Because the MAV will be the first non-terrestrial atmospheric launch vehicle mission ever attempted, it is highly desirable to perform integrated MAV system tests using the principle of test-as-you-fly." The relatively thin Martian atmosphere at MOLA aeroid, zero relative surface elevation, and the atmospheric density profile presents a challenge to replicate terrestrially. It is also essential to demonstrate vehicle stability, control rates, and control authority. Because the gravity on Earth is significantly greater than on Mars, the staging sequence, e.g. coast period durations, cannot be replicated, and the 2nd stage maneuver will still be in atmosphere for the terrestrial test.

Several elements of the integrated MAV test can be validated by launching the MAV from a high altitude platform. Some potential options include dropping the MAV from an aircraft, delivering the MAV to altitude with a sounding rocket, or launching off of a high altitude balloon platform. To understand the logistics, safety challenges, and potential implementation approaches, NASA's Balloon Program completed a study for the ISPT project to evaluate the potential of a balloon based altitude MAV launch.

\section{Balloon Launch}

The purpose of the Mars Ascent Vehicle (MAV) Balloon test is to loft a propulsion system on a balloon to above 20 $\mathrm{km}$. The propulsion system will be ignited and fly a demonstration flight. This will allow the propulsion system development team to simulate system performance in flight environments resembling the MAV Martian trajectory. A flight at these altitudes will provide an approximation of the dynamic pressure for critical events including the burn out of the first stage, separation, second stage ignition, and controllability. This proposal approach attempts to define the process to assess how a balloon flight test can be safely and effectively accomplished to satisfy this MAV system test. Recommendations are also made to improve the success of the test flight.

The concept of using a balloon as the first stage" or to get a rocket motor to a specific altitude and then to launch a rocket has been discussed for many years. Numerous general technical papers have been written on this subject. A few actual simplified attempts to do this have been made, but were not up to the rigorous level required by NASA for both Safety and flight operations. This means that there is little heritage that one can rely on from a practical conceptual implementation perspective. The conceptual approach for achieving this mission was to use as many of the established processes, procedures, guidelines, and approaches that are currently in place for balloon operations, rocket handling/integration, ground safety, and flight safety. The proposed conceptual approach attempts to meld the best and applicable pieces of each of these discipline areas to accomplish the desired mission. Figure 6 presents an overview of the proposed test operation. 


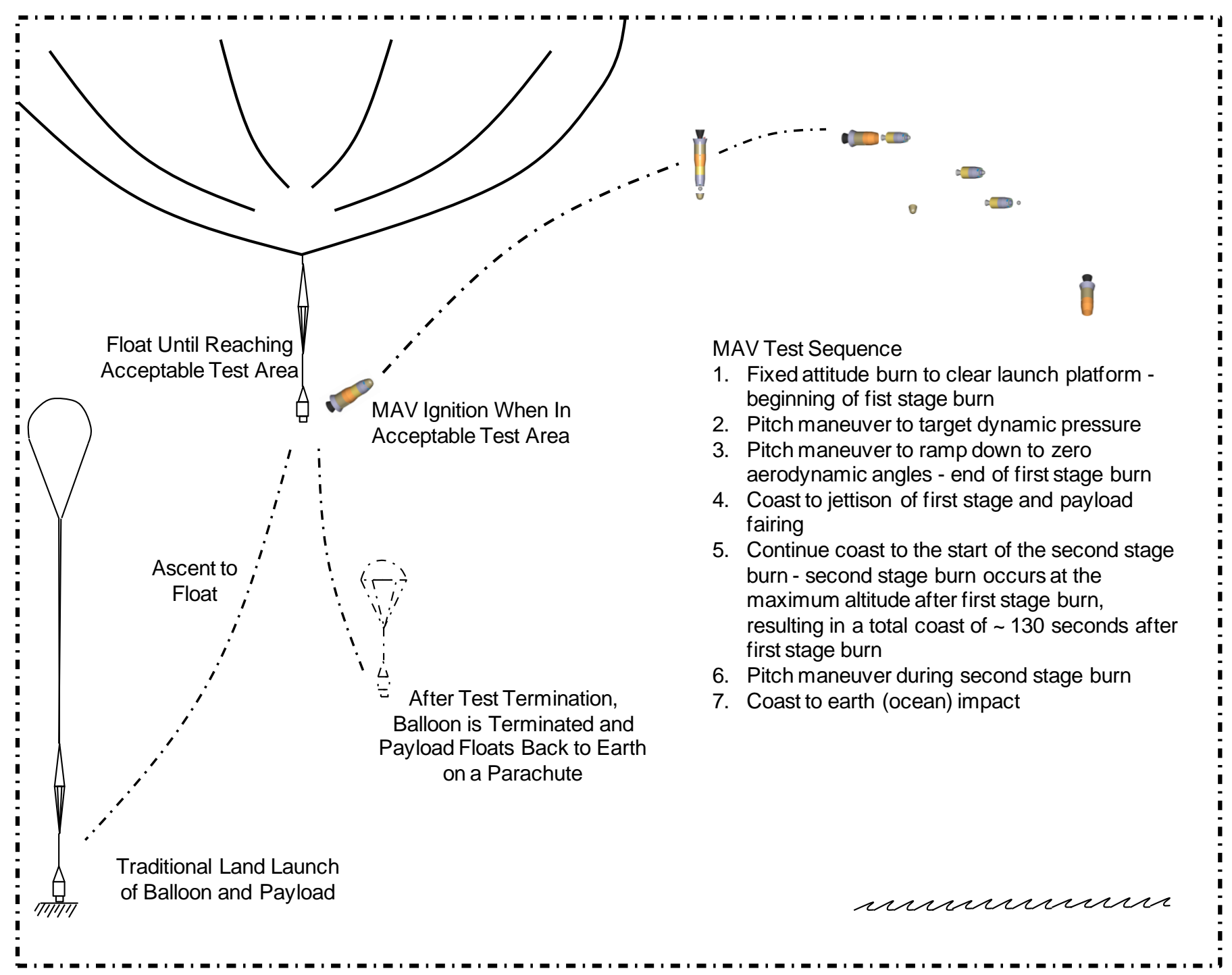

Figure 6 - Notional balloon launched MAV test sequence.

After launch, the balloon and payload will ascend to the desired float altitude. The entire system will be allowed to settle into a level flight. The balloon will be allowed to fly until it reaches the appropriate safe testing range. The balloon will then be vented to bring it down to the desired altitude, or ballasted up to the desired altitude and then stabilized at the desired altitude. The NASA Solar Pointing System will be used to orient the gondola to the desired firing azimuth. The payload location will be confirmed that the system is in a location acceptable from a Safety standpoint to initiate the test. System checks will be completed before initiating the firing sequence. With approval from flight safety, verification of altitude and orientation, and positive system checks, the propulsion system test will begin.

The propulsion test system will be enabled and initiated by ground command. The test plan has the propulsion system communication operating in a transmit mode only. For the baseline approach, telemetry from the propulsion system will be received at the balloon gondola and then transmitted back to the ground using the NASA Support Instrumentation Package (SIP) instrumentation and telemetry systems. The flight data would also be stored onboard. Nominally the MAV propulsion system would follow the following test sequence:

- Fixed attitude burn to clear launch platform beginning of first stage burn

- Pitch maneuver to target dynamic pressure

- Pitch maneuver to ramp down to zero aerodynamic angles - end of first stage burn

- Coast to jettison of first stage and payload fairing

- Continue coast to the start of the second stage burn - second stage burn occurs at the maximum altitude after first stage burn, resulting in a total coast of 130 seconds after first stage burn

- Pitch maneuver during second stage burn

- Coast to earth (ocean) impact 
The propulsion system flight test is expected to last several minutes. At the conclusion of the test, the test article will fall and impact in the ocean. The balloon flight system will be terminated per standard operating procedures via ground command. The balloon will be terminated and descend on its own. The payload will descend separately on a parachute.

Analysis was completed to characterize the ability to capture the key aerodynamic flight test parameters expected during the ascent through the relatively thin Martian atmosphere. Simulations were performed to match Q, while not exceeding Q-Alpha and simulating the control rates required for the Mars Ascent. A parameter sweep revealed a close match between the values of maximum $Q$ at an altitude of $27.5 \mathrm{~km}$. Figure 6 illustrates the agreement between the high altitude Earth environment and the Mars environment that can be achieved. The two cases are in agreement within less than one percent.

Results also indicated that $\mathrm{Q}$ is relatively insensitive to the launch elevation. This is important, as it allows Q-Alpha, which is very sensitive to launch elevation, to be targeted independently. The simulation results indicated that a launch elevation of $10^{\circ}$ from horizontal can match the expected Mars environment within one percent. A simulation of the Mars environment and comparison to the terrestrial test $\mathrm{Q}$ and Q-Alpha is provided in Figures 7 and 8

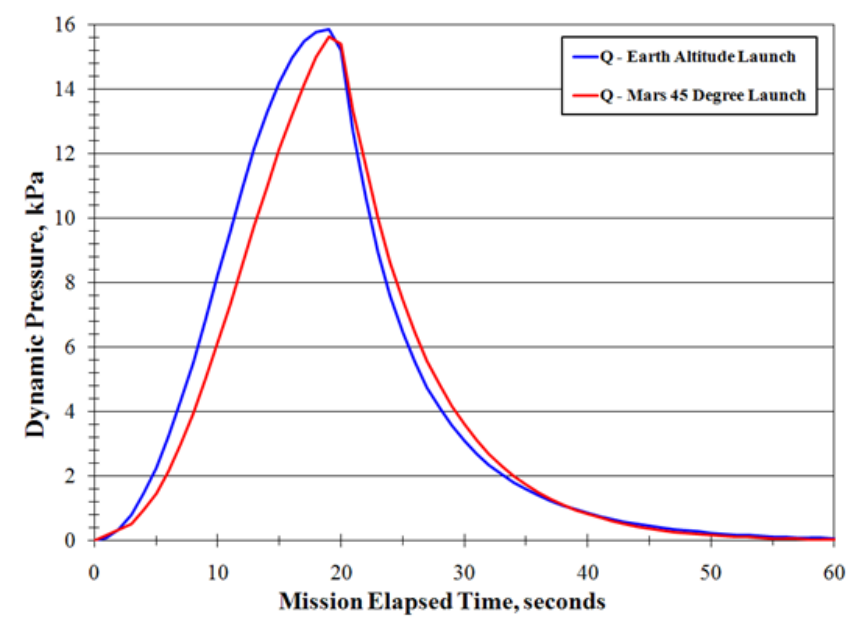

Figure 7 - Dynamic pressure comparison from balloon launch and Mars surface launch.

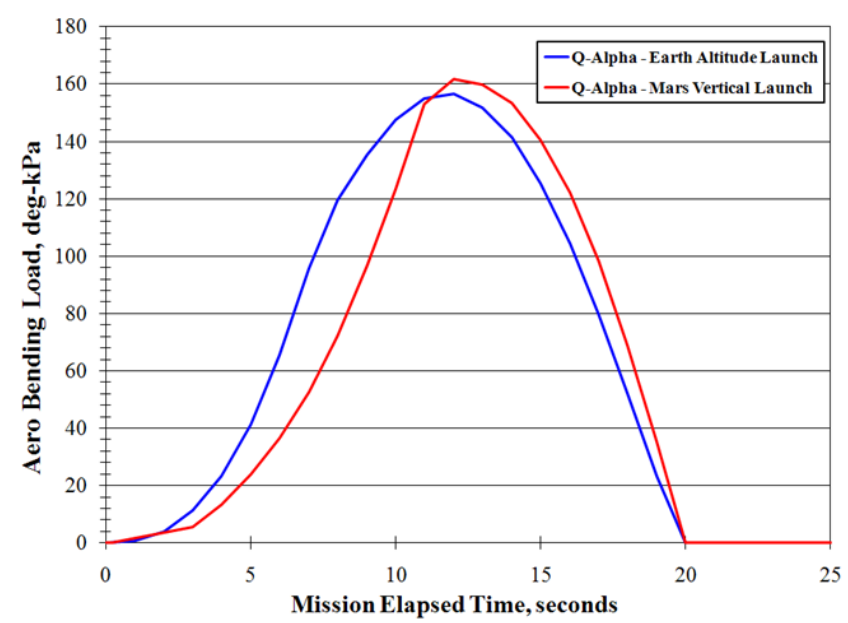

Figure 8 - Q-Alpa comparison from balloon launch and Mars surface launch.

The specific balloon chosen for a mission is primarily determined by the total suspended mass of the system, the desired mission float altitude, and the required flight duration. The NASA Balloon Program utilizes a family" of standard balloon designs to meet customers" requirements. Standard balloon performance is shown in Figure 9. Typical science missions desire to fly above a particular altitude, and the science users tend to want to fly as high as possible. This is why the majority of the standard balloon designs have float altitude in the 33.5 to $39.6 \mathrm{~km}(110,000$ to 130,000 foot) range. This mission is targeting a float altitude of $27.5 \mathrm{~km}(\sim 90,000 \mathrm{ft})$; this would require a 0.88 to a 2.6 Million Cubic Foot (MCF) balloon. This is small compared to the standard NASA balloon designs.

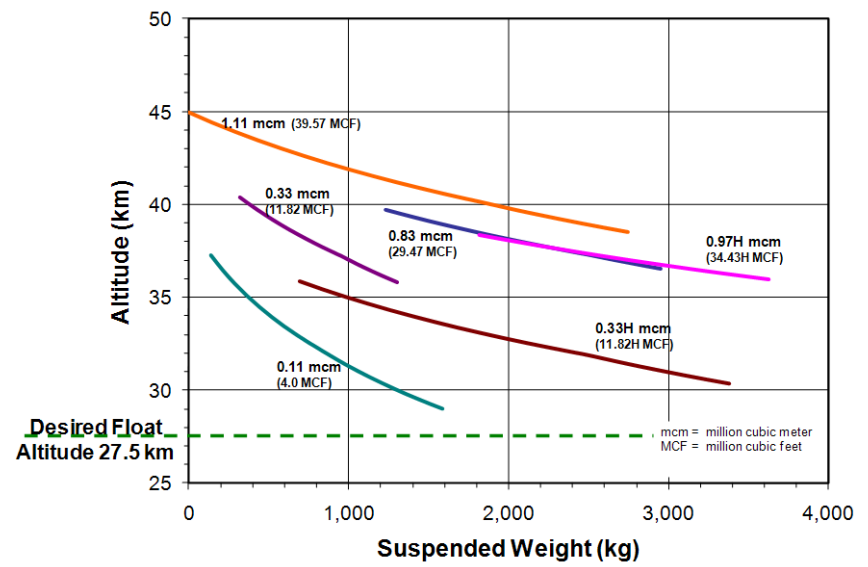

Figure 9 - Performance of standard NASA balloon designs.

The suspended mass of the system is usually a combination of two factors; the specific instrument or test system mass, and the mass of the required support systems (structure, electronics, protection systems, flight train components, etc.) Typically one tries to minimize the total suspended system mass to make the required balloon as small as possible, and 
the launch operations easier. In this case, there is an additional element to be considered as part of the system mass. The total suspended mass must also provide adequate stability for the initial thrust of the propulsion system. Stability of the system translates into additional system mass.

The type of flight profile desired is also a factor in selecting the balloon. The target profile desired to select proper balloon can be either a short duration or a longer duration flight. For reference, each of these is presented below.

- $\quad$ Short flight

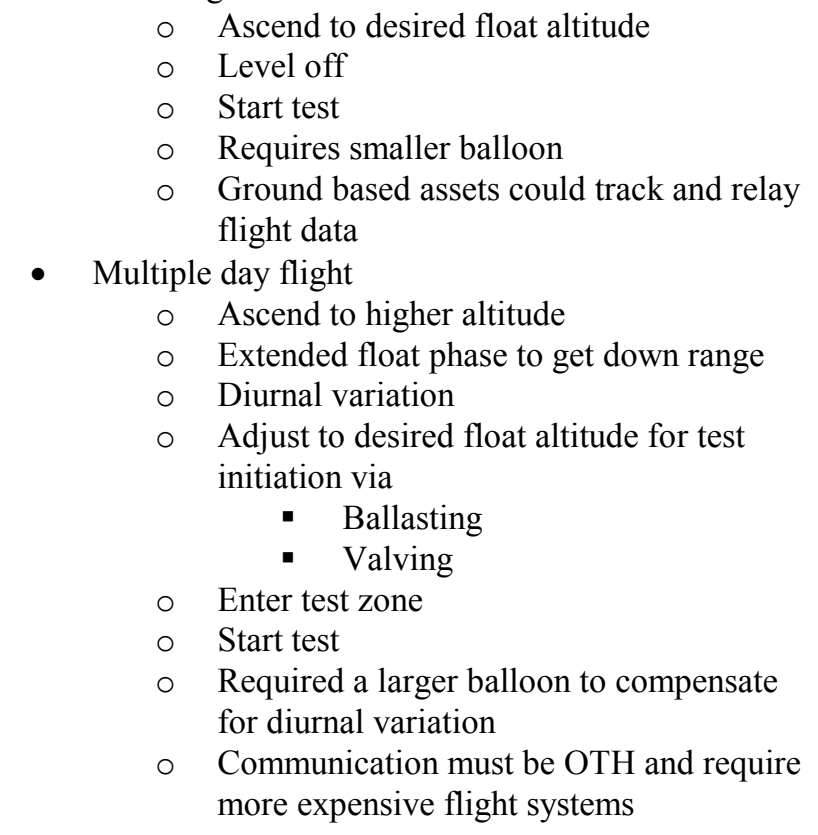

The single most significant constraint is the lack of a Flight Termination System (FTS) in combination with the expected range of the MAV. A FTS is a system used to destroy the propulsion system if it goes off course. This system can be used to prevent the flight system from entering a hazard space or leaving the defined test range. FTS's come in various forms that are tailored to the specific propulsion system. The number of locations in the world where this system can be flown without a FTS are quite limited. The lack of an FTS and expected range also eliminates the potential of a short duration flight.

Range safety dictates that without an FTS and with a guided propulsion system, the safety range must be the maximum energy footprint achievable. The maximum energy range case for the baseline earth launch was determined by assuming a highly unlikely scenario where a worst-on-worst failure for both stages would result in a maximization of down range on each stage. The resulting maximum energy downrange distances at various launch altitudes are presented in Figure 10. For the solid MAV design, maximum energy downrange distances were tracked for both the first stage impact and the second stage impact. The results indicate that for the baseline case, the impact for the first stage is on the order of $550 \mathrm{~km}$ and the impact for the second stage is $1460 \mathrm{~km}$.

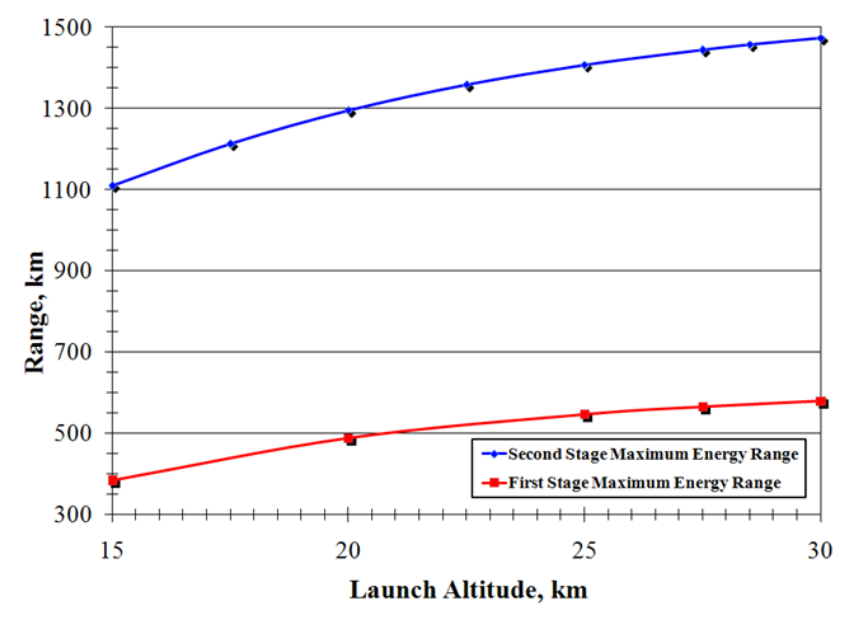

Figure 10 - Maximum range distances vs. launch
altitude.

The two stage range places significant constraints on the mission. If the mission were equipped with a Flight Termination System, the balloon would be allowed to ascend to the desired float altitude, and then the test initiated shortly thereafter. This mission would require a smaller balloon. Without the FTS and using the eontainment approach" for flight safety, the balloon flight time to get downrange to the acceptable test range is much longer. This requires the mission to be flown similar to the standard NASA Long Duration Balloon (LDB) missions and will dictate different flight hardware and a different balloon.

This mission can be accomplished with significant flight heritage support systems. Flight systems are required for communication, ballasting, valving, and balloon flight termination. The mission is recommended to use the Standard Instrumentation Package (SIP). The SIP is TDRSS/Iridium SIP uses an Octagon Pentium computer for Comm1 and Comm2 with over-the-horizon capabilities. A pair of heritage ballast hopper is also recommended with nearly $600 \mathrm{~kg}$ of ballast divided between the hoppers for redundancy. A Solar Pointing System (SPS) is also a heritage design to point the payload to a desired azimuth. The heritage system has a capability of $+/-5^{\circ}$, but $+/-1^{\circ}$ capability is expected prior to the MAV test. Valving can be performed with a standard EV-13 Helium Valve, and the mission will terminate with the proven Universal Terminate Package (UTP).

Using the desired mission profile to meet the science objectives, it is recommended this mission will use one of the standard NASA balloons in inventory in an LDB mode. An 11.82 million cubic foot balloon is recommended as the baseline. This balloon design has the following characteristics: 
Volume:

Nominal Payload:

Nominal Altitude:

Balloon Weight:

Inflated Dimensions:

Gore Length:

Number of Gores:

$11.82 \mathrm{MCF}$

5,500 pounds

102,900 feet

$\sim 3050$ pounds

Height: 228.8 feet

Diameter: 317.6 feet

431.48 feet

119

The 11.82 Heavy balloon has an excellent historical performance history. It can fly for an extended diurnal duration flight. As noted above, the balloon can accommodate up to 5,500 pounds suspended load. A detailed system mass has not been completed, but the final system mass would include both ballast and dead weight to increase the overall payload mass to provide stability for the MAV launch.

Assuming a 30 knot wind, it will take $\sim 27$ hours to get down range. The target altitude is $\sim 27.5 \mathrm{~km}$. A multiple day flight approach would follow the following pattern:

- Ascend to higher altitude - nominally $32.1 \mathrm{~km}$ during the day

- Extended float phase to get down range

- Diurnal variation - low altitude of $\sim 25.45 \mathrm{~km}$ at night

- Adjust to desired float altitude via valving in this case - two scenarios shown

- Valving during night portion of the flight

- Valving after reaching float altitude the second day

- Enter test zone (note - this could be during the day or night)

- Initiate test

There are numerous flight paths that can be taken to get to the desired target altitude at the desired time. The actual balloon flight performance will have to be analyzed before flight to ensure the proper desired flight profile. The balloon requires approximately 4 hours to achieve float altitude. The balloon then requires another 23 hours to meet the range safety constraints. Because of the diurnal heating conditions, the balloon can either be vented from above or drop ballast from a lower altitude to arrive at the target altitude for MAV deployment. Representative flight profiles are shown in Figure 11; using either venting or ballast to achieve the target altitude and the nominal flight path.

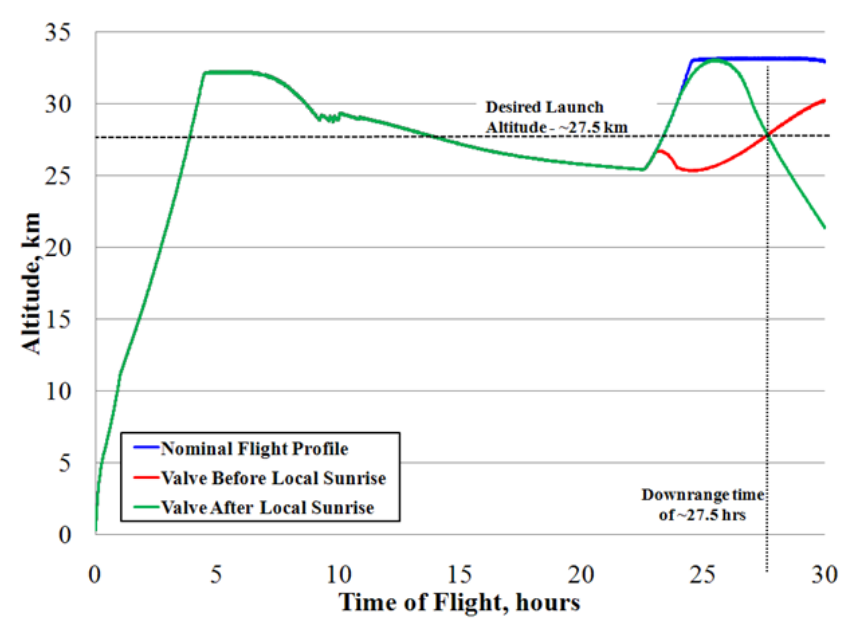

Figure 11 - Potential balloon flight profiles.

Based on the required range and the prevailing wind speeds, there are few options for a balloon launch of the MAV. Figure 12 illustrates the potential MAV deployment sites. This locations are extremely remote and provide logistic and communication challenges. The three highest rated launch sites are Vandenberg AFB, the Pacific Mission Range Facility at Barking Sands, Hawaii, and the Balloon Launching Facility at Alice Spring, Australia. All of these sites require a land launch and then a long traverse to arrive at the initial conditions. Based on winds analysis for mean wind velocity and direction and standard deviation, in additional to logistic challenges, the recommended launch site is Vandenberg.

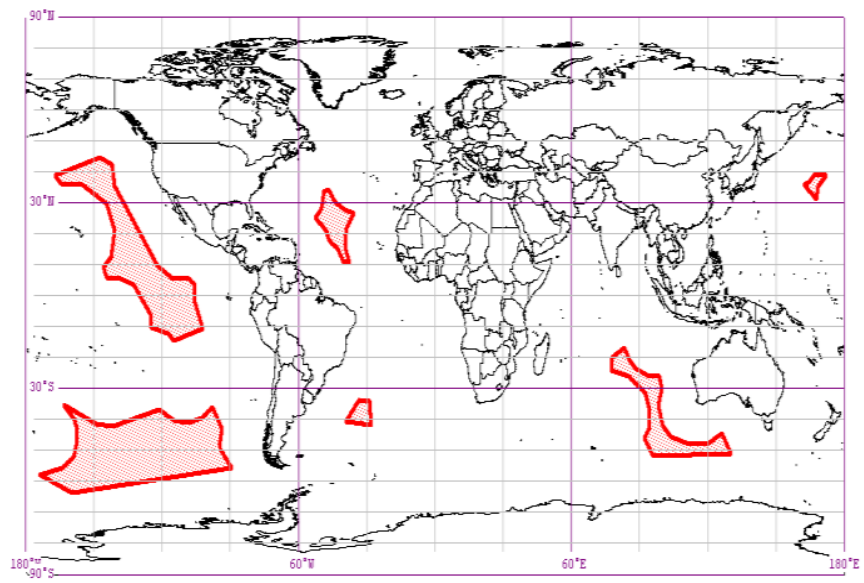

Figure 12 - Potential MAV deployment locations.

Detailed surface wind and float level wind analyses have not been completed, but Vandenberg is located at almost the same latitude as Ft. Sumner, NM where hundreds of scientific balloon flights have been launched. The upper level stratospheric winds at this latitude are well known. The desire would be to fly during the time of the year when the strongest winds are prevalent that would push the balloon to 
the West. This flight window, estimated at six weeks, is in July to mid August. The average wind speeds at the desired float altitude are on the order of 40 knots with a standard deviation of 6 to 8 knots. In simple terms, this would result in a higher likelihood of the balloon going downrange as desired to get to the test initiation point.

VAFB possesses a full complement of command, telemetry, radar tracking, and range control assets. There are facilities suitable for explosive ordnance storage, payload and MAV system assembly and processing. There is a substantial airfield that can be used for balloon launch operations. Specific climbout trajectories for VAFB were not run due to the time constrains for this proposal. These can be run to determine and refine the potential launch options. Another advantage of VAFB is that it is a domestic launch site which reduces the logistics challenges, travel costs, and significantly reduces the cost of Helium.

Overall, there appears to be options for a balloon based high altitude launch of the MAV. The balloon launch constraints are significantly driven by a lack of a flight termination system and therefore the maximum energy range. There would be considerable logistic and communication challenges associated with a balloon based MAV test. The balloon element costs are estimated at \$3M per launch and there would only be a potential launch window for six weeks out of every year.

\section{Ground Test Options}

A large number of a test plan objectives can be achieved through ground based testing as well. Ground based tests could either be fixed test stands with hardware in the loop system validation or an integrated ground launch. Static test fire options are used routinely to validate subsystem performance. Flight like electronics, commanded guidance, TVC actuation rates and generated torques, etc. can all be measured and tested within Mars simulated environments. A significant number of the MAV flight system risks can be retired through fixed asset ground tests.

The White Sands Test Facility (WSTF), for example, can simulate Martian atmospheric conditions for either solid or liquid based MAV propulsion systems. The WSTF can also condition propellants and test articles down to $4^{\circ} \mathrm{C}$; below the potential launch condition of the MAV.

If it was acceptable to deviate from the Mars atmospheric parameters, a surface launched MAV test can also be performed. A quick look was evaluated for a MAV flight test conducted out of White Sands, NM. For these simulations, the trajectory was optimized to minimize the aerodynamic loads, as they will far exceed the expected Martian environment. The values for Mars and earth based aerodynamic loads expected are shown in Table 1. The results show that the aerodynamic loads are significantly higher for a surface altitude test from White Sands. A liquid based, or optimal (Mars) thrust, system will experience double the Mars $\mathrm{Q}_{\text {Max }}$ and the baseline high thrust solution will be almost an order of magnitude higher. Loads analysis needs to be completed on the MAV to see if designed the MAV for the higher terrestrial test requirements will drive the mass of the MAV.

\section{Table 1 - Comparison to surface launch aerodynamic} loads

\begin{tabular}{|l|c|c|}
\hline \multicolumn{1}{|c|}{ Vehicle Design } & Max W, psf & $\begin{array}{c}\text { Max Q-Alpha, } \\
\text { psf-deg }\end{array}$ \\
\hline Mars Baseline & 457 & 54 \\
\hline Earth - solid & 3,939 & 381 \\
\hline Earth - Liquid & 993 & 672 \\
\hline
\end{tabular}

A terrestrial surface launch will have a distinct advantage in minimizing the MAV test range. For a two-stage test, the maximum ranges are $325 \mathrm{~km}$ and $518 \mathrm{~km}$ for the high thruster and optimal thrust solutions respectively. If it was acceptable to only test the first stage performance, MAV control, and separation events without a second stage burn, the maximum range can be reduced to only $50 \mathrm{~km}$. This would be a very practical range for multiple flight tests. The shorter range also significantly simplifies the communication with the MAV; any surface based test could leverage ground based assets for communication and should not require high altitude or satellite communication relay. The altitude and range vs. time for a White Sands ground based launch for only the first stage is shown in Figure 13.

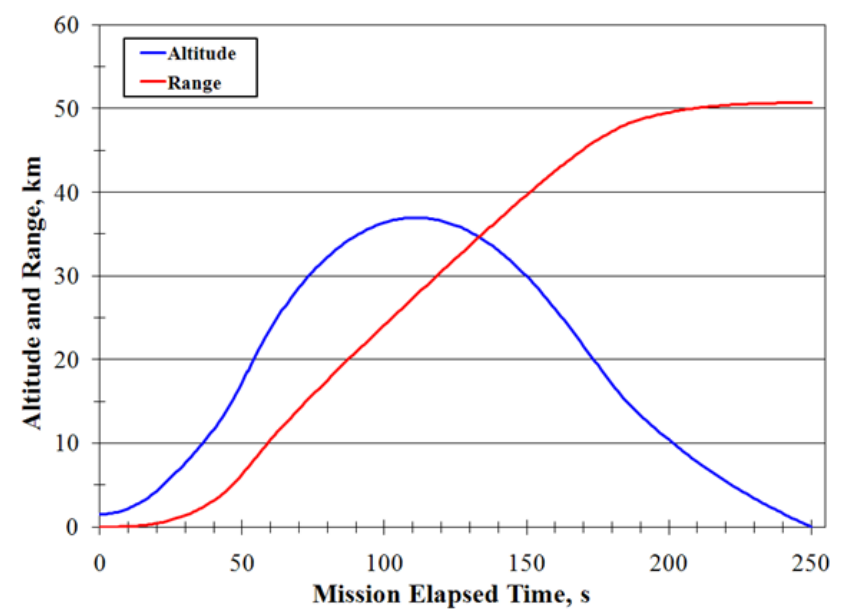

Figure 13 - Altitude and range for a ground based single stage test from the WSTF.

\section{Test Matrix}

The goal of any critical flight element is to test-as-you-fly and also fly-as-you-test. This presents a series of challenges for the MAV. There is no method to meet this objective entirely with a terrestrial launch, either at high altitude or ground based. A high altitude launch can better replicate the 
Mars environments, but at a higher cost. It is important to balance the additional knowledge gained from higher complexity testing with alternative risk mitigation strategies. The test options with validation fidelity are estimated in Table 2 .

\section{Notional Test Sequence}

For the MAV tests, the sequence of events will ideally complete the propellant loading of the MAV as expected for the MSR mission, including any planetary protection procedures. The MAV will then undergo simulated Earth launch and Mars EDL environments, experience some steadstate duration within the expected Mars surface environment; e..g low temperature storage, and then complete the MAV terrestrial flight demonstration.

Table 2 - Test matrix and validation fidelity

\begin{tabular}{|l|l|l|l|l|l|}
\cline { 2 - 5 } \multicolumn{1}{c|}{} & $\begin{array}{c}\text { Fixed Ground- } \\
\text { Based Testing }\end{array}$ & $\begin{array}{c}\text { Single-Stage } \\
\text { Ground- } \\
\text { Launch Test }\end{array}$ & $\begin{array}{c}\text { Two-Stage } \\
\text { Ground- } \\
\text { Launch Test }\end{array}$ & $\begin{array}{c}\text { Single-Stage } \\
\text { Altitude- } \\
\text { Launch Test }\end{array}$ & $\begin{array}{c}\text { Two-Stage } \\
\text { Altitude- } \\
\text { Launch Test }\end{array}$ \\
\hline Structural Loads & & Higher than expected & Higher than expected & \\
\hline First-Stage Performance & & & & \\
\hline Second-Stage Performance & & & & \\
\hline Separation Event & & & & \\
\hline RCS Performance & & & & \\
\hline TVC Performance & & & & \\
\hline GN\&C Performance & & & & \\
\hline Telemetry Performance & & & & \\
\hline Communication Performance & & & & \\
\hline
\end{tabular}

\section{Conclusions}

The MAV is one of the highest risk elements of the Mars Sample Return flagship mission. The ISPT project has initiated MAV system designs and subsystem technology development. The MAV has unique test challenges to testas-you-fly." High altitude options exist to closely simulate aerodynamic loads, but with limited flight opportunities, and logistic, communication, and safety challenges. Fixed ground test and surface launch options exist for lower fidelity system validation, but with easier implementation options. The ground based tests may also result in a vehicle design requirements greatly exceeding the actual Mars mission resulting in unnecessary mass penalties. The baseline recommendation remains the high altitude balloon test, but detailed operations and communication systems need to be assessed before a viable and practical test can be initiated. The ISPT project is continuing to explore MAV terrestrial flight system validation options for eventual MSR mission risk reduction.

\section{REFERENCES}

[1] Dux, I. J., Huwaldt, J. A., McKamey, R. S., and Dankanich, J. W., Mars Ascent Vehicle Gross Lift-off Mass Sensitivities for Robotic Mars Sample Return," 2011 IEEE Aerospace Conference, Big Sky, MT, March 5-12, 2011.

[1] Franklin, S. F., Slonski Jr., J. P., Kerridge, S., Noreen, G., Riedel, J. E., Stosic, D., Racho, C., Edwards, B., Austin, R., and Boroson, D., The 2009 Mars Telecom Orbiter Mission," 2005 IEEE Aerospace Conference, Big Sky, MT, March 2005.

[3] Dankanich, J. W., Mars Ascent Vehicle Technology Planning," 2009 IEEE Aerospace Conference, Big Sky, MT, March 2009.

[4] Stephenson, D., Mars Ascent Vehicle - Concept Development," AIAA-2002-4318, 38th Joint Propulsion Conference, July 2002.

[5] Way, D. W, et al., "Mars Science Laboratory: Entry, Descent, and Landing System Performance," 2007 IEEE Aerospace Conference, Big Sky, MT, March 2007. 


\section{BIOGRAPHIES}

John Dankanich is a Gray Research contractor to the NASA Glenn Research Center. He is the MAV technology development lead and electric propulsion lead systems engineer for the ISPT project. He has supported propulsion system development, Mars Ascent Vehicle design, lunar lander guidance simulations, planetary defense studies, and advanced propulsion design and testing. John has a B.S. in Physics and Aerospace Engineering and an M.S. in Aerospace Engineering from Purdue University.

Henry M. Cathey, Jr. is a NMSU, Physical Science Laboratory Engineering Group Supervisor and contractor to the NASA Wallops Flight Facility. He has been involved in all aspects of the NASA Balloon Program for over 15 years including balloon design, fabrication, performance analysis, balloon model testing, field support and integration, balloon flight operations, and the NASA Super Pressure Balloon development. Henry received his B.E. in Mechanical Engineering from Vanderbilt University and his M.S in Mechanical Engineering, with a concentration in Space Systems, from the George Washington University. Henry is a past Chair of the American AIAA Balloon Systems Technical Committee.
Ian Dux is an Aerospace Engineer in the Mission Design and Systems Analysis Branch at the NASA Glenn Research Center. His areas of expertise include trajectory optimization and systems analysis for both earth-to-orbit and in-space vehicle systems. Ian holds a B.S. Mechanical Engineering from the Pennsylvania State University and is currently working on an M.S. in Aerospace Engineering from the Georgia Institute of Technology.

David A. Smith is a Wyle, Systems Engineer contractor supporting the Mission Design and Systems Analysis Branch at the NASA Glenn Research Center. He specializes in trajectory optimization and simulation as well as 6-DOF vehicle simulation code and tool development. Dave has a B.A. in Physics from West Virginia University and a Ph.D. in Atmospheric, Earth and Ocean Sciences form Old Dominion University. 